\title{
Free-Radical Copolymerization VII. Reinterpretation of Velocity-of-Copolymerization Data
}

\author{
Takeshi FuKudA, Yung-Dae MA, ${ }^{*}$ Keiji KuBO, ${ }^{* *}$ \\ and Akihiko TAKADA \\ Institute for Chemical Research, Kyato University, \\ Uji, Kyoto 611, Japan
}

(Received June 1, 1989)

\begin{abstract}
Velocity-of-copolymerization data available in the literature were reinterpreted on the basis of the current notions that the penultimate-unit effect with respect to absolute values of propagation rate constant (but not with respect to the monomer reactivity ratios) is a general rule, and that the termination step is diffusion-controlled, i.e., normal. By making a few simplifying approximations, a new velocity equation was derived, which was found to describe experimental data generally better than the classical equation based on the terminal propagation model with a single adjustable parameter $\phi$, the cross-termination factor. The single adjustable parameter $s$ included in the new equation, which measures the penultimate-unit effect, was found to have a strong correlation with the monomer reactivity ratios such that the smaller $r_{1} r_{2}$, the smaller is $s$, i.e., the more significant is the penulimate-unit effect. This result is in support of the relation $r_{1} r_{2}=s_{1} s_{2}$ suggested by the phenomenological theory [T. Fukuda et al., Makromol. Chem., Rapid Commun., 8, 495 (1987)].
\end{abstract}

KEY WORDS Copolymerization Velocities / Penultimate Model / Normal

Termination / Single-Parameter Equation / Penultimate Effect and $r_{1} r_{2} /$

In former times, experimental data on copolymerization velocities were analyzed, in almost all cases, by assuming that the terminalmodel propagation scheme ${ }^{1}$ was correct.
Among the classical velocity equations along this line, the following one commonly termed Walling's equation ${ }^{2}$ has been most widely used:

$$
\omega=\frac{r_{1} f_{1}^{2}+2 f_{1} f_{2}+r_{2} f_{2}^{2}}{\left[\left(r_{1} f_{1} / \omega_{1}\right)^{2}+2 \phi\left(r_{1} r_{2} f_{1} f_{2} / \omega_{1} \omega_{2}\right)+\left(r_{2} f_{2} / \omega_{2}\right)^{2}\right]^{1 / 2}}
$$

with

$$
\begin{aligned}
& \omega=k_{\mathrm{p}} / k_{\mathrm{t}}^{1 / 2} \\
& \omega_{i}=k_{\mathrm{p} i} / k_{\mathrm{ti}}^{1 / 2} \quad(i=1 \text { or } 2) \\
& \phi=k_{\mathrm{t} 12} /\left(k_{\mathrm{t} 1} k_{\mathrm{t} 2}\right)^{1 / 2}
\end{aligned}
$$

where $k_{\mathrm{p}}$ and $k_{\mathrm{t}}$ are the rate constants of propagation and termination, respectively, with the subscripts 1 and 2 referring to the homopolymerizations, $r_{i}$ 's are the monomer reactivity ratios, and $f_{i}$ 's are the feed monomer compositions. This equation allows the determination of the cross-termination factor $\phi$ without knowing $k_{\mathrm{t} 1}$ and $k_{\mathrm{t} 2}$. This factor should be close to unity unless $1-2$ crossterminations are chemically favored over 1-1 and 2-2 homo-terminations. Values of $\phi$ experimentally determined via eq 1 were often

* Present address: Department of Chemical Engineering, Dankook University, Seoul 140, Korea.

** Present address: Kuraray Co., Ltd., 2045-1 Sakatsu, Kurashiki, Okayama 710, Japan. 


\section{T. FUKUDA et al.}

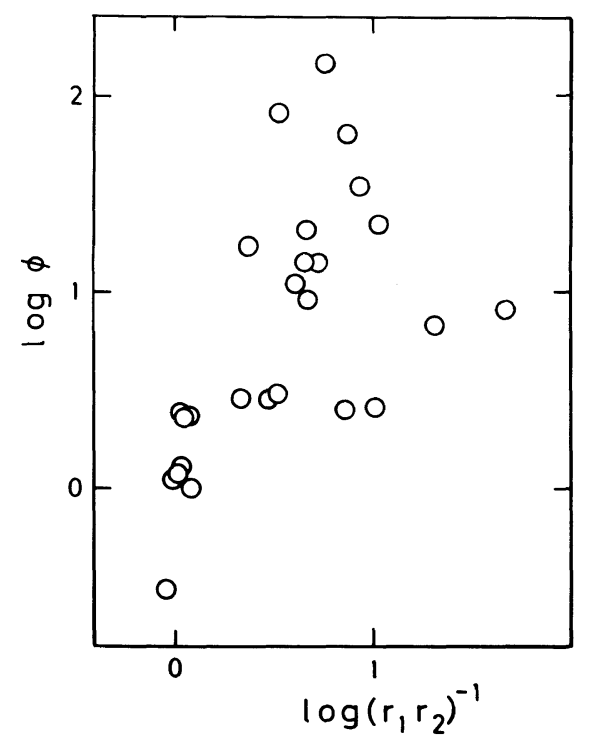

Figure 1. Values of $\phi$ for various copolymerization systems plotted against $r_{1} r_{2}$ (see Table II and the text for details).

composition-dependent and, in a large majority of systems, much larger than unity, as Figure 1 shows (see below for details).

Recently, we showed that the bulkcopolymerization of styrene (ST) and methyl methacrylate (MMA) fails to conform to the terminal model with respect to absolute values of $k_{\mathrm{p}},{ }^{3}$ and that a penultimate-unit effect is responsible for this phonomenon. ${ }^{4}$ Similar failure of the classical model has been observed also for $p$-chlorostyrene (pCS)/methyl acrylate (MA), ${ }^{5}$ ST/ethyl acrylate (EA) ${ }^{6}$ and MMA/ vinyl acetate (VA) ${ }^{6}$ systems, while the MMA/ butyl methacrylate (BMA) system, a copolymerization of homologous monomers, was found to conform to the terminal model. ${ }^{7}$ Also importantly, the absolute values of $k_{\mathrm{t}}$ for all these systems were found to be always between the $k_{\mathrm{t} 1}$ and $k_{\mathrm{t} 2}$ of the respective systems, thus showing no particular preference for crosstermination. This invalidates the previous estimates based on eq 1, i.e., $\phi \simeq 13$ for $\mathrm{ST} /$ MMA, 147 for $\mathrm{pCS} / \mathrm{MA}, 35$ for ST/EA, 10 for MMA/VA and 1.1 for MMA/BMA.

These current results decisively support the notion of diffusion-controlled termination in copolymerization as well as in homopolymerization, ${ }^{8}$ and urge reinterpretation of velocityof-copolymerization data for other systems. Since diffusion-controlled termination models $^{7,8}$ are numerically close to the chemical model with $\phi=1$, we may assume this $\phi=1$ model simply for a practical purpose, and analyze velocity data for approximate information on the propagation steps. This work reports the results of such an analysis of the available literature data. Our main interest is to examine possible correlation between the monomer reactivity ratios and the penultimate model parameters $s_{1}$ and $s_{2}$ introduced previously. ${ }^{3}$ The relation

$$
r_{1} r_{2}=s_{1} s_{2}
$$

was suggested by our phenomenological treatment of the penultimate-unit effect considered to be of radical-stabilization-energetic origin..$^{9,10}$

\section{RATE EQUATION AND DATA ANALYSIS}

The penultimate-model expression for $k_{\mathrm{p}}$ reads $^{3}$

$$
k_{\mathrm{p}}=\frac{\bar{r}_{1} f_{1}^{2}+2 f_{1} f_{2}+\bar{r}_{2} f_{2}^{2}}{\left(\bar{r}_{1} f_{1} / \bar{k}_{11}\right)+\left(\bar{r}_{2} f_{2} / \bar{k}_{22}\right)}
$$

where $\bar{r}_{i}$ and $\bar{k}_{i i}$ are given by

$$
\begin{aligned}
& \bar{r}_{i}=\frac{r_{i}^{\prime}\left(r_{i} f_{i}+f_{j}\right)}{r_{i}^{\prime} f_{i}+f_{j}} \quad(i \neq j) \\
& \bar{k}_{i i}=\frac{k_{i i i}\left(r_{i} f_{i}+f_{j}\right)}{r_{i} f_{i}+s_{i}^{-1} f_{j}} \quad(i \neq j)
\end{aligned}
$$

with

$$
\begin{aligned}
& r_{i}=k_{i i i} / k_{i i j} \quad(i \neq j) \\
& r_{i}^{\prime}=k_{j i i} / k_{j i j} \quad(i \neq j) \\
& s_{i}=k_{j i i} / k_{i i i} \quad(i \neq j)
\end{aligned}
$$

In these relations, $k_{i j m}$ is the propagation rate constant with $i, j$ and $m$ representing the 
penultimate, terminal and monomer units, respectively ( $i, j, m=1$ or 2 ). Clearly, the penultimate model reduces to the terminal model when the equalities $r_{1}=r_{1}^{\prime}, r_{2}=r_{2}^{\prime}$, and $s_{1}=s_{2}=$ 1 hold simultaneously.

The chemically controlled termination model with $\phi=1$ can be expressed as

$$
k_{\mathrm{t}}^{1 / 2}=p_{1} k_{\mathrm{t} 1}^{1 / 2}+p_{2} k_{\mathrm{t} 2}^{1 / 2}
$$

where $p_{1}\left(=1-p_{2}\right)$ is the fraction of the polymer radicals with monomer unit 1 at the active end. This is given by

$$
\frac{p_{1}}{p_{2}}=\frac{\bar{r}_{1} f_{1} / \bar{k}_{11}}{\bar{r}_{2} f_{2} / \bar{k}_{22}}
$$

Combination of eq 6 and 12 along with the use of eq 13 gives the following expression for the steady-state polymerization factor $\omega=k_{\mathrm{p}} / k_{\mathrm{t}}^{1 / 2}$ :

$$
\omega=\frac{\bar{r}_{1} f_{1}^{2}+2 f_{1} f_{2}+\bar{r}_{2} f_{2}^{2}}{\left(\bar{r}_{1} f_{1} / \bar{\omega}_{1}\right)+\left(\bar{r}_{2} f_{2} / \bar{\omega}_{2}\right)}
$$

where

$$
\bar{\omega}_{i}=\frac{\omega_{i}\left(\bar{r}_{i} f_{i}+f_{j}\right)}{\bar{r}_{i} f_{i}+s_{i}^{-1} f_{j}} \quad(i \neq j)
$$

with $\omega_{i}$ being given by eq 3 or, in the present terminology, by

$$
\omega_{i}=k_{i i i} / k_{t i}^{1 / 2} \quad(i=1 \text { or } 2)
$$

On analyzing literature data, we make the following approximations or simplifications. First, we assume that $r_{i}=r_{i}^{\prime}\left(=\bar{r}_{i}\right)$ for all systems. In view of the fact that the composition curves of most systems are describable by the terminal model at least to a good approximation, this would be a permissible simplification for the present purpose of semiquantitative analysis.

Secondly, we set $s_{1}=s_{2}=s$. As a matter of fact, this would not be generally correct. One reason why we nevertheless dare to introduce this assumption becomes clear if we examine the structure of eq 14 (or eq 6). It can be mathematically shown that when $r_{1} r_{2}=1, s_{1}$ and $s_{2}$ cannot be uniquely determined. For example, when $r_{1}=r_{2}=1$, eq 14 may be re- written as

$$
\begin{gathered}
\left(\omega^{-1}-f_{1}^{2} \omega_{1}^{-1}-f_{2}^{2} \omega_{2}^{-1}\right) /\left(f_{1} f_{2}\right)= \\
\left(s_{1} \omega_{1}\right)^{-1}+\left(s_{2} \omega_{2}\right)^{-1}
\end{gathered}
$$

Clearly, the measurements of $\omega$ as a function of composition $f_{i}$ allow determination of the sum on the right hand side of eq 17 but not $s_{1}$ and $s_{2}$ separately. This means that as the product $r_{1} r_{2}$ becomes closer to one, the reliability of the estimated values of $s_{1}$ and $s_{2}$ and hence of the product $s_{1} s_{2}$, in which we are particularly interested, becomes poorer. It can happen that even when the true values of $s_{i}$ are close to one, small changes in experimental data result in almost any values of $s_{i}$ on the least-squares curve-fitting calculations. The assumption of $s_{1}=s_{2}$ frees us at least from this contradiction. Another reason for making this assumption is that the values of $r_{1} / \omega_{1}$ and $r_{2} /$ $\omega_{2}$ are often very different from each other. In such systems, the reliability of $s_{1}$, for example, is much lower than that of $s_{2}$ or vice versa, hence $s_{1} s_{2}$ of poor reliability. For example, the pCS(1)/MA(2) system has $r_{1} / \omega_{1}$ about 400 times as large as $r_{2} / \omega_{2}$. As is clear by examining eq $14-16$, it is virtually impossible to determine $s_{2}$ of this system. If we nevertheless carry out the least-squares calculations, we may formally obtain an "optimum" $s_{2}$, which obviously is totally unreliable. For systems in which $r_{1} r_{2}$ is not close to one and in which $r_{1} /$ $\omega_{1} \sim r_{2} / \omega_{2}$, the assumption is, of course, unnecessary. However, insofar as the product $s_{1} s_{2}$ is concerned, the results obtained with and without the assumption are not unreasonably different from each other in most cases, owing to partial cancellation between the first and second terms in the denominator in eq 14 . After all, we may mention that the single parameter $s\left(=s_{1}=s_{2}\right)$ will be reasonably close to either of the two parameters that makes a larger contribution to $\omega$ (eq 14). Since values of $s_{1}$ must be statistically scattered around values of $s_{2}$ and vice versa, the values of $s$ would correctly reflect the general trend, if 
data are sufficiently available. Also. attractive is the formal simplicity of eq 14 with the single adjustable parameter $s$. As will be seen later, most velocity data can be described with fair to sufficient accuracy by this single-parameter equation.

Last to be mentioned is that many authors do not report values of initiation rate $R_{i}$ or the relevant rate constant

$$
R_{i}=2 f^{\prime} k_{\mathrm{d}}[\mathrm{I}]
$$

where $[\mathrm{I}]$ is the initiator concentration, and the other symbols have conventional significance. Whenever the values of $2 f^{\prime} k_{\mathrm{d}}$ for the two homopolymerizations are available, we estimate the copolymerization values by assuming $2 f^{\prime} k_{\mathrm{d}}$ to be linear with composition. In other cases, we employ $K$ (and $K_{i}$ 's) in place of $\omega$ (and $\omega_{i}$ 's), where $K$ is the coefficient appearing in the familiar equation for the polymerization rate $R_{\mathrm{p}}$ :

$$
R_{\mathrm{p}}=K[\mathrm{I}]^{1 / 2}[\mathrm{M}]=\omega\left(2 f^{\prime} k_{\mathrm{d}}\right)^{1 / 2}[\mathrm{I}]^{1 / 2}[\mathrm{M}]
$$

This is equivalent to assuming $2 f^{\prime} k_{\mathrm{d}}$ to be constant. We exclude data which lack the $R_{\mathrm{p}}$ value(s) for one or both of the homopolymerizations. By these rules, we analyzed as many data as we know of. There is a fairly large number of relevant reports in the literature. However, many deal with the same pair of monomers with different solvents and/or at different temperatures. In such a case, we chose, rather arbitrarily, a representative re- port. The number of independent systems that we analyzed was about 25 , a rather unexpectedly small number. We are not quite confident, however, that this is all.

\section{RESULTS AND DISCUSSION}

We first simulated the ST(1)/MMA(2) bulk copolymerization $\mathrm{data}^{2}$ by the abovementioned equations of differing degree of approximation, i.e.,

$$
\begin{aligned}
& k_{\mathrm{p}}=\frac{r_{1} f_{1}^{2}+2 f_{1} f_{2}+r_{2} f_{2}^{2}}{\left(r_{1} f_{1} / \bar{k}_{11}\right)+\left(r_{2} f_{2} / \bar{k}_{22}\right)} \\
& \omega=\frac{r_{1} f_{1}^{2}+2 f_{1} f_{2}+r_{2} f_{2}^{2}}{\left(r_{1} f_{1} / \bar{\omega}_{1}\right)+\left(r_{2} f_{2} / \bar{\omega}_{2}\right)} \\
& K=\frac{r_{1} f_{1}^{2}+2 f_{1} f_{2}+r_{2} f_{2}^{2}}{\left(r_{1} f_{1} / \bar{K}_{1}\right)+\left(r_{2} f_{2} / \bar{K}_{2}\right)}
\end{aligned}
$$

with

$$
\begin{aligned}
& \frac{\bar{k}_{11}}{k_{111}}=\frac{\bar{\omega}_{1}}{\omega_{1}}=\frac{\bar{K}_{1}}{K_{1}}=\frac{r_{1} f_{1}+f_{2}}{r_{1} f_{1}+s_{1}^{-1} f_{2}} \\
& \frac{\bar{k}_{22}}{k_{222}}=\frac{\omega_{2}}{\omega_{2}}=\frac{\bar{K}_{2}}{K_{2}}=\frac{r_{2} f_{2}+f_{1}}{r_{2} f_{2}+s_{2}^{-1} f_{1}}
\end{aligned}
$$

The experimental values of $k_{\mathrm{p}}, \omega$, and $K$ are plotted against $f_{1}$ in Figures $2 \mathrm{a}, 2 \mathrm{~b}$, and $2 \mathrm{c}$, respectively. The dotted line in Figure $2 \mathrm{a}$ shows eq 20 computed with the best-fit values of $s_{1}$ and $s_{2}$ ( 0.30 and 0.53 , respectively), while the solid line in the same figure was computed under the above-mentioned restriction of $s_{1}=$

Table I. Comparison of the values of $s$ determined on the basis of the kinetic equations $\left(s_{1}=s_{2}=s\right)$ of differing degree of approximation

\begin{tabular}{lcccc}
\hline \multirow{2}{*}{ Experimental data } & \multicolumn{4}{c}{$s$} \\
\cline { 2 - 5 } & ST/MMA & ST/MMA/TOL & pCS/MA & MMA/BMA $^{\mathrm{c}}$ \\
\hline$k_{\mathrm{p}}$ (eq 20, rigorous) & 0.34 & 0.27 & 0.35 & 0.96 \\
$\omega$ (eq 21, approximate) & 0.33 & 0.32 & 0.43 & 1.22 \\
$K$ (eq 22, approximate) & 0.30 & 0.31 & 0.39 & - \\
\hline
\end{tabular}

a Styrene/methyl methacrylate at $40^{\circ} \mathrm{C}$ in bulk (ref. 3).

b Styrene/methyl methacrylate at $40^{\circ} \mathrm{C}$ in toluene (ref.4).

c $p$-Chlorostyrene/methyl acrylate in bulk at $40^{\circ} \mathrm{C}$ (ref. 5).

d Methyl methacrylate/butyl methacrylate in bulk at $30^{\circ} \mathrm{C}$ (ref. 7). 

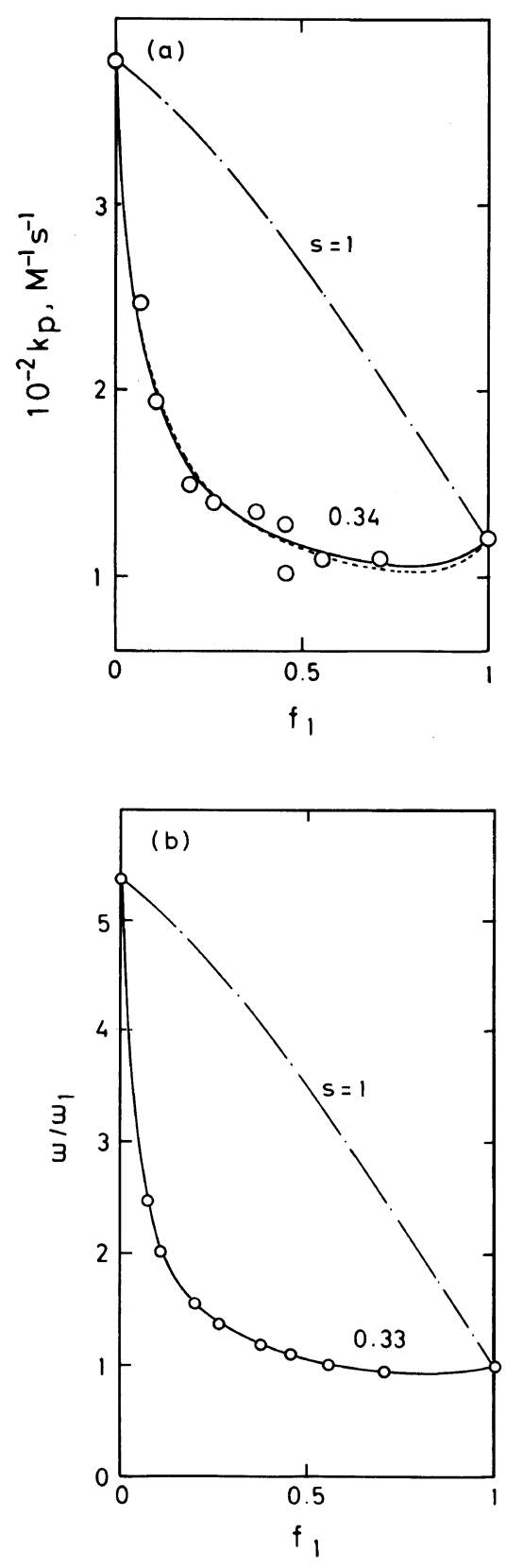

$s_{2}=s$, the best-fit value of $s$ being 0.34 . Seemingly, both calculative curves reproduce the experimental data nearly equally well. This means that at this level of experimental accuracy, it is difficult to evaluate the two parameters $s_{1}$ and $s_{2}$ individually with sufficient

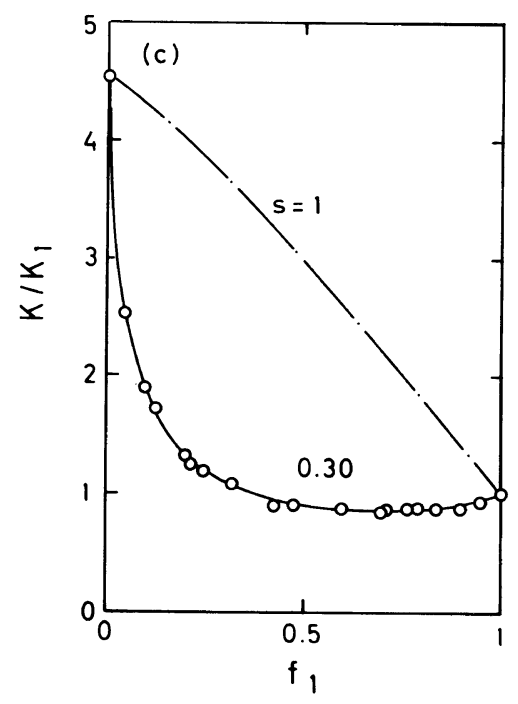

Figure 2. Comparison between experimental and calculative data for the bulk-copolymerization of ST and MMA at $40^{\circ} \mathrm{C}^{3}$ : (a) eq 20 with $s_{1}=0.30$ and $s_{2}=0.53$ (dotted curve) and eq 20 with $s\left(=s_{1}=s_{2}\right)=0.34$ (solid curve); (b) eq 21 with $s=0.33$ (solid curve); (c) eq 22 with $s=0.30$ (solid curve).

accuracy, as already mentioned. The curve is particularly insensitive to $s_{2}$, which accordingly will be accompanied by a large error. On the other hand, the curve with the restriction of $s_{1}=s_{2}$ is sensitive enough to the single parameter $s$. This is also the case with the $\omega v s$. $f_{1}$ (Figure $2 \mathrm{~b}$ ) and $K$ vs. $f_{1}$ (Figure $2 \mathrm{c}$ ) curves, from which we obtain best-fit values of $s$ of 0.33 and 0.30 , respectively. We made similar analysis with the $\operatorname{pCS}(1) / \mathrm{MA}(2)$ bulkcopolymerization data. ${ }^{5}$ Also for this system, the values of $s$ estimated with the $k_{\mathrm{p}}, \omega$, and $K$ data reasonably agree with each other (Table I). Again, it is indicated that eq 21 and 22 may be reasonable approximations. (As reported previously, ${ }^{11}$ the composition curve of the pCS/MA system is better represented by the penultimate model than by the terminal model. Hence, it is expected that better agreement between the calculative and experimental curve and a more accurate value of $s$ will be obtained by removing the approximation of $\bar{r}_{i}=r_{i}=$ constant that we made in this paper.) 
Table II. Results of curve-fitting analyses of velocity data ${ }^{a}$

\begin{tabular}{|c|c|c|c|c|c|c|c|}
\hline System & \multirow{2}{*}{$r_{1}$} & \multirow[b]{2}{*}{$r_{2}$} & \multirow{2}{*}{$s$} & \multirow{2}{*}{$(\sigma, \%)$} & \multirow{2}{*}{$\phi$} & \multirow{2}{*}{$(\sigma, \%)$} & \multirow{2}{*}{ ref } \\
\hline $\mathbf{M}_{1} / \mathbf{M}_{2} / \mathbf{T}\left({ }^{\circ} \mathrm{C}\right) /$ Solv. & & & & & & & \\
\hline $\begin{array}{l}\mathrm{ST} / \mathrm{MMA} / 40 / \text { Non } \\
\quad\left(\text { from } k_{\mathrm{p}}\right)\end{array}$ & 0.523 & 0.460 & $\begin{array}{c}0.33 \\
(0.34)\end{array}$ & $(1.1)$ & 13 & $(18.0)$ & 3 \\
\hline $\begin{array}{l}\mathrm{ST} / \mathrm{MMA} / 40 / \mathrm{TOL} \\
\quad\left(\text { from } k_{\mathrm{p}}\right)\end{array}$ & 0.534 & 0.393 & $\begin{array}{c}0.32 \\
(0.27)\end{array}$ & $(5.7)$ & 14 & $(22.2)$ & 4 \\
\hline $\begin{array}{l}\mathrm{pCS} / \mathrm{MA} / 40 / \text { Non } \\
\quad\left(\text { from } k_{\mathrm{p}}\right)\end{array}$ & 1.21 & 0.144 & $\begin{array}{c}0.43 \\
(0.35)\end{array}$ & $(7.2)$ & 147 & $(18.0)$ & 5 \\
\hline $\begin{array}{l}\text { MMA/BMA/30/Non } \\
\quad\left(\text { from } k_{\mathrm{p}}\right)\end{array}$ & 0.79 & 1.27 & $\begin{array}{c}0.96 \\
(1.22)\end{array}$ & ( 1.4$)$ & 1.1 & $(1.5)$ & 7 \\
\hline $\mathrm{ST} / \mathrm{EA} / 40 /$ Non & 0.781 & 0.171 & 0.63 & $(11.7)$ & 45 & $(14.3)$ & 6 \\
\hline MMA/VA/40/TOL & 22.7 & 0.013 & 0.55 & $(6.0)$ & 10 & $(7.5)$ & 6 \\
\hline $\mathrm{ST} / \mathrm{PFS} / 60 /$ Non & 0.43 & 0.22 & 0.72 & $(3.0)$ & 2.6 & $(3.0)$ & 12 \\
\hline ST/EMA/60/Non & 0.53 & 0.41 & 0.35 & $(2.5)$ & 14 & $(14.1)$ & 13 \\
\hline ST/iBMA/60/Non & 0.55 & 0.40 & 0.35 & $(5.6)$ & 21 & $(18.8)$ & 13 \\
\hline ST/nNMA/60/Non & 0.52 & 0.80 & 0.34 & $(7.6)$ & 17 & ( 7.6$)$ & 13 \\
\hline ST $/ \mathrm{BA} / 60 /$ Non & 0.76 & 0.15 & 0.67 & $(5.0)$ & 35 & (9.3) & 14 \\
\hline ST/TCP/60/Non & 7.8 & 0.017 & 0.29 & $(6.5)$ & 63 & ( 3.9$)$ & 15 \\
\hline $\mathrm{ST} / \mathrm{mVPh} / 60 /$ Non & 0.91 & 1.21 & 1.55 & $(3.0)$ & 0.31 & $(5.3)$ & 16 \\
\hline $\mathrm{ST} / \alpha \mathrm{VN} / 60 / \mathrm{Non}$ & 0.67 & 1.35 & 0.63 & $(1.5)$ & 2.4 & ( 2.9) & 17 \\
\hline $\mathrm{ST} / \mathrm{DEF} / 60 /$ Non & 0.3 & 0.07 & 0.45 & $(7.3)$ & 8.1 & $(13.8)$ & 18 \\
\hline $\mathrm{ST} / 1-\mathrm{CB} / 70 /$ Non & 0.274 & 1.68 & 0.80 & $(11.5)$ & 2.9 & $(10.6)$ & 19 \\
\hline ST/MAN/60/Non & 0.3 & 0.16 & 0.49 & $(15.9)$ & 6.7 & $(7.0)$ & 20 \\
\hline $\mathrm{ST} / \mathrm{pMS} / 60 /$ Non & 1.16 & 0.82 & 0.95 & $(2.7)$ & 1.2 & $(2.6)$ & 21 \\
\hline PFS/MMA/60/Non & 0.90 & 0.98 & 0.66 & ( 6.9$)$ & 2.3 & $(4.6)$ & 12 \\
\hline pMS/MMA/60/Non & 0.32 & 0.29 & 0.27 & $(6.6)$ & 22 & $(13.7)$ & 16 \\
\hline MMA/DMA/30/EAc & 0.719 & 1.268 & 0.9 & $(5.4)$ & 1.3 & ( 4.9$)$ & 7 \\
\hline MMA/AN/60/DMF & 1.2 & 0.15 & 0.3 & $(4.9)$ & 14 & $(7.4)$ & 22 \\
\hline $\mathrm{AN} / \mathrm{MA} / 60 / \mathrm{DMF}$ & 0.67 & 1.26 & 0.72 & $(8.3)$ & 2.3 & $(11.8)$ & 23 \\
\hline $\mathrm{AN} / \alpha \mathrm{CMA} / 60 / \mathrm{DMF}$ & 0.15 & 2.00 & 0.68 & $(10.4)$ & 3 & ( 8.4$)$ & 23 \\
\hline $\mathrm{AN} / \alpha \mathrm{BrMA} / 60 / \mathrm{DMF}$ & 0.081 & 4.2 & 0.72 & $(10.3)$ & 2.8 & $(12.1)$ & 23 \\
\hline VC/VDC/50/Non & 0.22 & 3.8 & 0.94 & $(8.1)$ & 1.0 & $(8.4)$ & 24 \\
\hline
\end{tabular}

a Abbreviations: $\mathrm{AN}=$ acrylonitrile; $\alpha \mathrm{CMA}=\alpha$-chloro-methylacrylate; $\alpha \mathrm{BrMA}=\alpha$-bromo-methylacrylate; $\alpha \mathrm{VN}=\alpha$ vinylnaphthalene; $\mathrm{BA}=n$-butylacrylate; $\mathrm{BMA}=n$-butyl methacrylate; 1 - $\mathrm{CB}=1$-chlorobutadiene; $\mathrm{DEF}=$ diethyl fumarate; $\mathrm{DMA}=n$-dodecyl methacrylate; $\mathrm{DMF}=$ dimethylformamide; $\mathrm{EA}=$ ethyl acrylate; $\mathrm{EAc}=$ ethyl acetate; $\mathrm{EMA}=$ ethyl methacrylate; iBMA= iso-butyl methacrylate; $\mathrm{MAN}=$ methacrylonitrile; $\mathrm{MA}=$ methyl acrylate; $\mathrm{MMA}=$ methyl methacrylate; $\mathrm{mVPh}=m$-vinylphenol; $\mathrm{nNMA}=n$-nonyl methacrylate; $\mathrm{PFS}=$ pentafluorostyrene; $\mathrm{pCS}=p$-chlorostyrene; $\mathrm{pMS}=p$-methoxystyrene; $\mathrm{ST}=$ styrene; $\mathrm{TCP}=3,3,3$-trichloropropene; $\mathrm{TOL}=$ toluene; $\mathrm{VA}=$ vinyl acetate $\mathrm{VC}=$ vinyl chloride $\mathrm{VDC}=$ vinylidene chloride.

The results of the curve-fitting analysis of the literature data with eq 21 (or eq 22) are summarized in Table II. As a measure of agreement between theory and experiment, we also give in the table the value of $\sigma(\%)$ defined by

$$
\sigma=100 v^{1 / 2}
$$

with

$$
v=(1 / n) \sum_{i=1}^{n}\left[\left(X_{i, \exp }-X_{i, \mathrm{cal}}\right) / X_{i, \mathrm{cal}}\right]^{2}
$$

where $X_{i, \exp }$ and $X_{i, \text { cal }}$ are the experimental and calculative values of $X(=\omega$ or $K)$ for the $i$ th experimental point out of a total of $n$ such points. For comparison, we analyzed the same data on the basis of the classical equation 1. As the values of $\sigma$ show, the present $s$-equations generally give a better fit to the experiments 


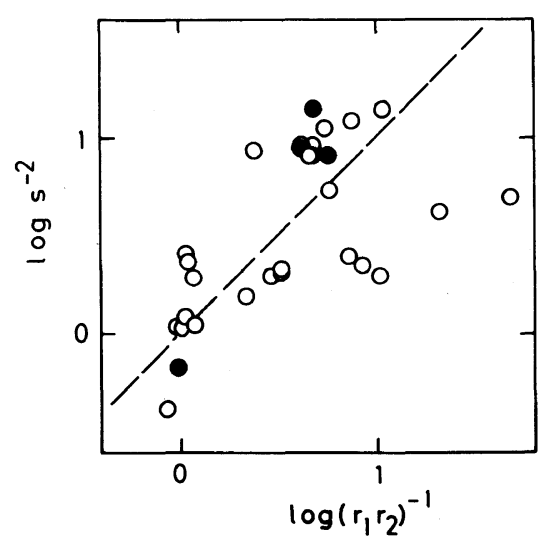

Figure 3. Plot of $s^{-2} v s .\left(r_{1} r_{2}\right)^{-1}$ for various copolymerization systems. The filled circles are relevant to the $k_{\mathrm{p}}$ data. The broken line shows the relations $s^{-2}=\left(r_{1} r_{2}\right)^{-1}$ indicated previously. ${ }^{9}$

rather than the classical $\phi$-equation (see Table II). For some systems such as ST/MAN, AN/ $\alpha C M A$ and $A N / \alpha B r M A$, eq 21 (or eq 22) is a poor approximation (see Table II for the abbreviations). These and possibly some more systems may require more detailed studies about the propriety of the present model.

In Figure 3, the best-fit values of $s$ are plotted as a function of $r_{1} r_{2}$. Evidently, a positive correlation exists between $s^{-2}$ and $\left(r_{1} r_{2}\right)^{-1}$, i.e., the smaller is $r_{1} r_{2}$, the smaller is $s^{2}$. More importantly, these data seem to be consistent, or at least not in conflict, with the relation 5 suggested by our phenomenological theory. ${ }^{9}$ Hence these velocity data may be understood as being in support of our basic postulate that the penultimate unit generally has a significant effect on the stability and hence the reactivity of a polymer radical..$^{9,10}$

To summarize, we have reinterpreted velocity-of-copolymerization data in terms of the penultimate-propagation model by postulating that the termination step be normal, i.e., $\phi=1$. The proposed velocity equation (the $s$-equation) describes the experimental data available in the literature generally better than the classical $\phi$-equation. A positive correlation exists between $r_{1} r_{2}$ and $s$, indicating that the smaller is $r_{1} r_{2}$, the more significant is the penultimate-unit effect with respect to absolute values of propagation rate constants (but not necessarily with respect to the monomer reactivity ratios $r_{1}$ and $r_{2}$ ).

\section{REFERENCES}

1. (a) F. R. Mayo and F. M. Lewis, J. Am. Chem. Soc., 66, 1954 (1944); (b) T. Alfrey, Jr. and G. Goldfinger, J. Chem. Phys., 12, 205 (1944); (c) F. T. Wall, J. Am. Chem. Soc., 66, 2050 (1944); (d) I. Sakurada, "Kojugo-Hanno," Society of Polymer Chemistry, Japan, Tokyo (1944).

2. C. Walling, J. Am. Chem. Soc., 71, 1930 (1949).

3. T. Fukuda, Y.-D. Ma, and H. Inagaki, Macromolecules, 18, 17 (1985).

4. T. Fukuda, K. Kubo, Y.-D. Ma, and H. Inagaki, Polym. J., 19, 523 (1987).

5. Y.-D. Ma, T. Fukuda, and H. Inagaki, Macromolecules, 18, 25 (1985).

6. Y.-D. Ma and T. Fukuda, to be published.

7. K. Ito and K. F. O'Driscoll, J. Polym. Sci., Polym. Chem. Ed., 17, 3913 (1979).

8. J. N. Atherton and A. M. North, Trans. Faraday Soc., 58, 2049 (1962).

9. T. Fukuda, Y.-D. Ma, and H. Inagaki, Makromol. Chem., Rapid Commun., 8, 495 (1987).

10. T. Fukuda and Y.-D. Ma, to appear in Macromolecules.

11. T. Fukuda, Y.-D. Ma, and H. Inagaki, Polym. J., 14, 705 (1982).

12. W. A. Pryor and T. L. Huang, Macromolecules, 2, 70 (1969).

13. A. K. Chaudhuri and S. R. Palit, Makromol. Chem., 121, 33 (1969).

14. J. H. Bradbury and H. W. Melville, Proc. R. Soc. London, Ser. A, 222, 456 (1954).

15. J. Szafko, E. Turska, and G. P. Lapatina, Makromol. Chem., 176, 2981 (1975).

16. E. P. Bonsall, L. Valentine, and H. W. Melville, Trans. Faraday Soc., 48, 763 (1952).

17. S. Lashaek and E. Broderic, J. Polym. Sci., 39, 241 (1959).

18. C. Walling and E. A. McElhill, J. Am. Chem. Soc., 73, 2819 (1951).

19. S. Kohjiya, M. Kurokawa, K. Iwata, Y. Imoto, T. Enoki, and S. Yamashita, Polym. J., 17, 661 (1985).

20. K. Ito, J. Polym. Sci., Polym. Chem. Ed., 16, 2725 (1978).

21. E. P. Bosall, L. Valentine, and H. W. Melville, $J$. Polym. Sci., 7, 39 (1951).

22. J. Szafko, cited in O. Prochaza, and P. Kratochvil, $J$. Polym. Sci., Polym. Chem. Ed., 21, 3269 (1983).

23. J. Szafko and E. Turska, Makromol. Chem., 156, 311 (1972).

24. K. Matsuo and W. H. Stockmayer, Macromolecules, 10, 658 (1977). 